\title{
Forensic age estimation in living adolescents with CT imaging of the clavicula-impact of low-dose scanning on readers' confidence
}

\author{
Sebastian Gassenmaier ${ }^{1} \cdot$ Juergen F. Schaefer ${ }^{1} \cdot$ Konstantin Nikolaou $^{1} \cdot$ Michael Esser $^{1} \cdot$ Ilias $_{\text {Tsiflikas }}{ }^{1}$
}

Received: 21 February 2020 / Revised: 15 April 2020 / Accepted: 16 July 2020 / Published online: 28 July 2020

(C) The Author(s) 2020, corrected publication 2021

\begin{abstract}
Objectives Computed tomography (CT) imaging of the clavicula displays the reference standard for forensic bone age diagnostics in adolescents and young adults. Consequently, highest efforts on radiation reduction are warranted. Therefore, the aim of this study was to investigate the feasibility of low-dose (LD) CT imaging of the clavicula for age estimation in living adolescents.

Methods A total of 207 non-contrast chest CT of 144 patients born between 1988 and 2012, performed in 2018 due to various clinical indications, were included in this retrospective study. The mean patient age was $16.9 \pm 6.6$ years. Patients were divided into a LD $(n=146)$ and standard-dose (SD; $n=61)$ group. Image quality, confidence levels, and ossification stages (using the 5stage classification including the subgroups $2 \mathrm{a}-3 \mathrm{c}$ ) were assessed by two radiologists independently. Radiation dose was determined via dosimetry software.

Results Dose simulation with z-axis reduction to depict the clavicula only resulted in a median exposure of $0.1 \mathrm{mSv}$ (IQR: $0.0)$ in LD compared with $0.9 \mathrm{mSv}$ (IQR: 0.6$)$ in $\mathrm{SD}(p<0.001)$. The median image quality was rated by both readers significantly worse in LD compared with SD on a Likert scale ranging from 1 to 4 with a median of 3 (IQR: 1) versus 4 (IQR: $0 ; p<0.001$ for both readers). There was an almost perfect agreement for the ossification stages between both readers with a Cohen's kappa of $0.83(p<0.001)$. Median confidence levels of both readers were not significantly different between LD and SD in the decisive subgroups $2 \mathrm{a}-3 \mathrm{c}$.

Conclusions Low-dose CT imaging of the clavicula for age estimation in living adolescents is possible without loss of readers' confidence.

Key Points

- Radiological bone age diagnostics in young delinquents with unknown exact chronological age is important as the judicial systems differentiate between youths and adults.

- Low-dose computed tomography scanning of the medial clavicular joint for forensic age estimation is feasible in living adolescents without loss of readers' confidence.

- Sufficient image quality of the medial clavicular joint for forensic bone age diagnostics in living adolescents is achievable using a median dose of $0.1 \mathrm{mSv}$.
\end{abstract}

Keywords Forensic medicine - Multidetector computed tomography · Age determination by skeleton · Radiation dosage · Sternoclavicular joint

Ilias Tsiflikas

ilias.tsiflikas@med.uni-tuebingen.de

1 Department of Diagnostic and Interventional Radiology, University Hospital Tuebingen, Hoppe-Seyler-Straße 3,

72076 Tuebingen, Germany

\section{Abbreviations}

CT Computed tomography

CTDIvol Computed tomography dose index

DLP Dose length product

ED Effective dose

ICRP 103 International Commission on Radiological Protection 103

IQR Interquartile range 


$\begin{array}{ll}\text { LD } & \text { Low-dose } \\ \text { MRI } & \text { Magnetic resonance imaging } \\ \text { SD } & \text { Standard-dose }\end{array}$

\section{Introduction}

$\mathrm{X}$-ray imaging of the left hand for bone age estimation in living subjects using the atlas of Greulich and Pyle displays a standard procedure in radiology [1]. However, this method is connected with a certain margin of error in adolescents after fully completed skeletal development of the left hand [1-3]. Therefore, other methods involving X-ray or magnetic resonance imaging (MRI) of the wrist, pelvis, and knee have been introduced by several authors for more reliable age estimation [4-7]. The current radiological reference standard for forensic age estimation in living adolescents is displayed by computed tomography (CT) imaging of the medial clavicular epiphysis [3, 8-10]. This method was firstly introduced by Kreitner et al in 1997 using a four-stage classification for age estimation [9]. In later studies, a fifth stage was appended for more precise age estimation [11]. Although in some investigations conventional radiography of the medial clavicular epiphysis was applied, several studies pointed out that the data provided by CT imaging is superior [12-14]. Due to the technical development of CT scanners including thin slice acquisition, the five-stage classification was further edited by Kellinghaus et al with the introduction of more detailed subgroups $2 \mathrm{a}-\mathrm{c}$ and $3 \mathrm{a}-\mathrm{c}[15$, $16]$.

Forensic age estimation in living adolescents is carried out for medicolegal issues only, mostly due to severe criminal actions and the resulting juridical implications. Therefore, greatest efforts are required to reduce radiation dose as the imaging does not contribute to the diagnosis of a disease in these young delinquents. The ALARA (as low as reasonably achievable) principle should always be observed.

Therefore, the aim of this study was to investigate the feasibility of low-dose (LD) CT imaging of the clavicula for forensic age determination of living adolescents and its impact on image quality as well as on the confidence level of the reader.

\section{Materials and methods}

\section{Study characteristics}

This monocentric, retrospective study was approved by the institutional review board. All CT examinations of the chest which were performed in our institution in 2018 were searched via the radiology information system for the subgroup of patients born between 1988 and $2012(n=338)$. Patients born prior to 1988 or after 2012 were excluded due to expected complete skeletal maturation or fully immature stage of ossification. In a next step, all contrast-enhanced examinations were excluded to achieve homogeneity of the study group, resulting in 232 non-contrast examinations. Imaging studies which did not depict the complete thorax including the medial sternoclavicular joint were also removed from the cohort. This resulted in the final study group of 207 examinations in 144 patients (Fig. 1).

\section{Imaging protocols and dose evaluation}

The CT examinations were performed using three different scanners (Siemens SOMATOM Force, Siemens SOMATOM Definition Flash, Siemens SOMATOM Definition AS+; all Siemens Healthineers). Imaging was conducted in supine position and in cranio-caudal scanning direction with a collimation of $0.6 \mathrm{~mm}$ after scout acquisition. Two different protocols were used for LD imaging: the first protocol consisted of a tube voltage of $100 \mathrm{kV}$ with tin filtration and a reference tube current of $96 \mathrm{mAs}$ using automated adaptation of tube current (CARE Dose; Siemens Healthineers); the second protocol involved automated adaptation of tube voltage and tube current (CARE kV and CARE Dose; Siemens Healthineers), using a reference tube voltage of $100 \mathrm{kV}$ without tin filtration and a reference tube current of $10 \mathrm{mAs}$. For standard-dose (SD) imaging, a tube voltage of 90-120 kV without tin filtration and a reference tube current of 55$123 \mathrm{mAs}$ depending on the scanner were applied. Onemillimeter slice thickness and 1-mm increment applying a high-frequency (bone) kernel in transversal and coronal planes were used for image reconstruction. Iterative algorithms (SAFIRE or ADMIRE; Siemens Healthineers) were used as in clinical routine. Patients were divided into two different groups for comparison, according to the imaging protocol, which resulted in $146 \mathrm{LD}$ vs. 61 SD examinations.

Dose length product (DLP), computed tomography dose index $\left(\mathrm{CTDI}_{\mathrm{vol}}\right)$, and effective dose (ED) were evaluated using a commercially available dosimetry and tracking software (Radimetrics; Bayer Healthcare). Effective dose was calculated according to the International Commission on Radiological Protection 103 (ICRP 103). Additionally, a dose simulation with reduced $z$-axis scanning which involved the whole clavicula only was performed via this software.

\section{Image evaluation}

Image evaluation was performed independently by two radiologists with 1 year and 13 years of experience on a dedicated workstation (GE Centricity PACS RA 1000; General Electric Healthcare), respectively. Images were evaluated in axial and coronal planes using a slice thickness of $1 \mathrm{~mm}$. The presence of artifacts was noted using a nominal scale. Image quality was evaluated according to a Likert scale ranging from 1 
Fig. 1 Flowchart of this study

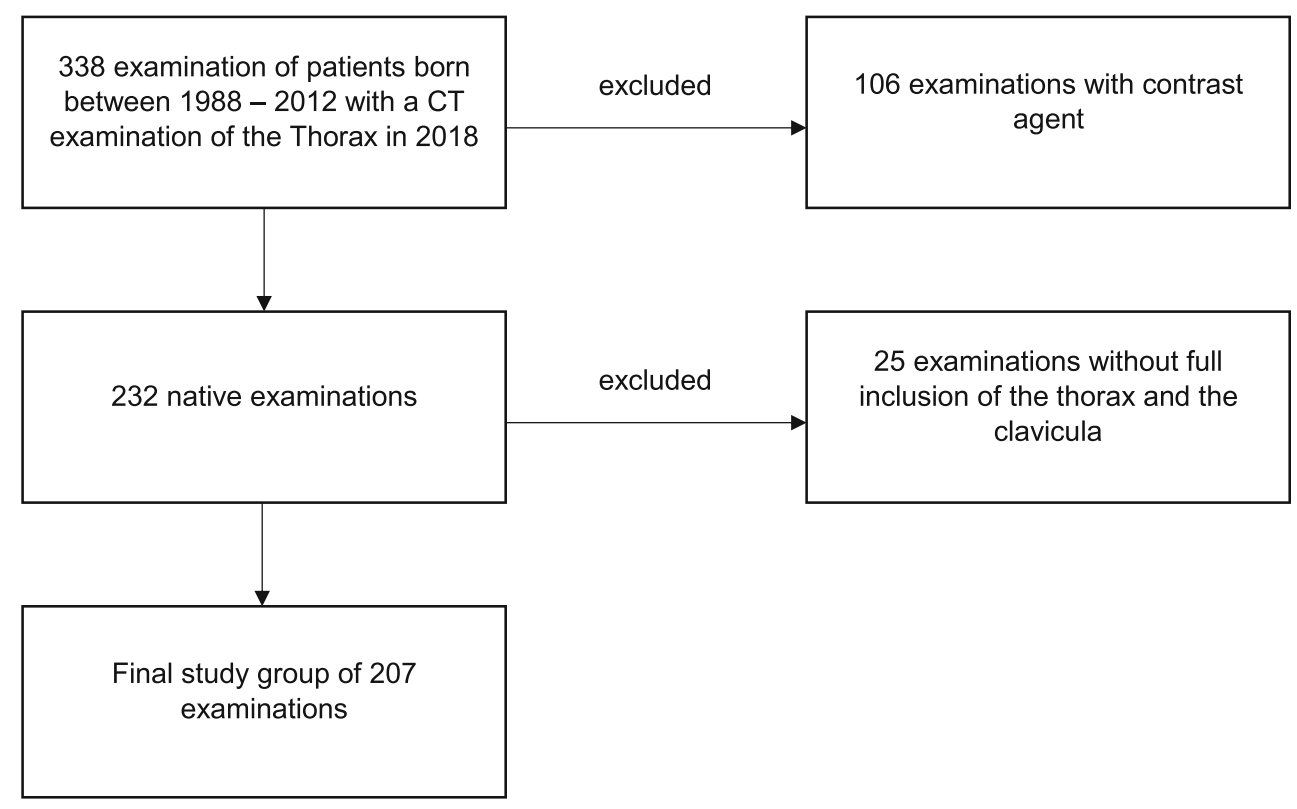

(non-diagnostic) to 4 (excellent). The previously published ossification stages by Schmeling et al (stages 1-5) and the further subclassification of Kellinghaus et al (2a, b, c and 3a, $b, c)$ were used for age estimation $[11 ; 15]$ :

Stage 1: epiphyseal ossification center not ossified Stage 2a: less than one-third of the ossification center ossified

Stage $2 \mathrm{~b}$ : less than two-thirds but more than one-third of the ossification center ossified

Stage 2c: more than two-thirds of the ossification center ossified

Stage 3a: less than one-third of the epiphyseal cartilage ossified

Stage $3 \mathrm{~b}$ : less than two-thirds but more than one-third of the epiphyseal cartilage ossified

Stage 3c: more than two-thirds of the epiphyseal cartilage ossified

Stage 4: full ossification with visible scar

Stage 5: full ossification without visible scar

In the case of two different stages between both medial clavicular joints within one patient, the higher stage was chosen. Additionally, each reader stated the confidence level of the age estimation on a Likert scale ranging from 1 (low) to 4 (very high). A subset of 32 pairs of examinations which were scanned twice within 100 days was used for intra-reader agreement.

\section{Statistical analysis}

Proprietary statistical software was used for evaluation (IBM SPSS Statistics, version 23; JMP 14, SAS Institute). Normal distribution of the variables was assessed with the ShapiroWilk test. The Student's $t$ test and the Wilcoxon signed ranksum test were used for independent parametric and nonparametric data. In case of paired data, the dependent $t$ test and the paired Wilcoxon signed rank test were applied. For unpaired and paired binominal data, the chi-square test and McNemar test were used, respectively. Spearman's correlation was applied for ordinal scaled units between both radiologists. Cohen's kappa was applied for inter- and intra-reader agreement. The significance level alpha was set at 0.05 .

Table 1 Characteristics of the study group

\begin{tabular}{ll}
\hline Characteristics & Values \\
\hline Examinations & $n=207$ (112 in male patients) \\
$\begin{array}{l}\text { Patients } \\
\text { Age at examination date }\end{array}$ & \\
Mean age \pm std. & $16.9 \pm 6.6$ years \\
Range & $5.4-29.8$ years \\
Low-dose group (LD) & \\
Number of examinations & $n=146$ \\
Mean age \pm std. & $13.7 \pm 4.7$ years \\
Range & $5.4-28.8$ years \\
Standard-dose group (SD) & \\
Number of examinations & $n=61$ \\
Mean age \pm std. & $24.5 \pm 3.4$ years \\
Range & $15.3-29.8$ years \\
Subset 2a-3c & \\
Number of examinations & $n=65$ (LD: $n=45 ;$ SD: $n=20)$ \\
Mean age \pm std. & LD: $17.1 \pm 2.1$ years; SD: $21.4 \pm 3.0$ years \\
Range & LD: $13.9-21.5$ years; SD: $15.3-29.8$ years \\
\hline
\end{tabular}


Table 2 Comparison of radiation dose between low-dose and standard-dose imaging

\begin{tabular}{llll}
\hline & Low-dose imaging & Standard-dose imaging & $p$ value \\
\hline DLP $(m G y * c m)$ CTDI $_{\text {vol }}(\mathrm{mGy})$ & 8.3 (IQR: 7.0$)$ & 226.8 (IQR: 68.5$)$ & $<0.001$ \\
Effective dose $(\mathrm{mSv})_{\text {Simulated effective dose }^{1}(\mathrm{mSv})}$ & 0.3 (IQR: 0.2) & 6.7 (IQR: 1.8$)$ & $<0.001$ \\
\end{tabular}

${ }^{1}$ Analysis of 55 low-dose and 50 standard-dose cases

$D L P$ dose length product, $C T D I_{v o l}$ computed tomography dose index

\section{Results}

\section{Patients' characteristics}

A total of 207 examinations were evaluated. The mean patient age was $16.9 \pm 6.6$ years (range, 5.4-29.8 years). A total of 112 examinations were performed in male patients. Further characteristics are displayed in Table 1.

\section{Radiation dose exposure}

Median DLP was significantly lower in LD scans with $8.3 \mathrm{mGy} * \mathrm{~cm}$ (interquartile range (IQR): 7.0) vs. $226.8 \mathrm{mGy}^{*} \mathrm{~cm}$ (IQR: 68.5) in SD scans $(p<0.001)$. Accordingly, median ED was also lower in LD scans with $0.2 \mathrm{mSv}$ (IQR: 0.1$)$ vs. $4.6 \mathrm{mSv}$ (IQR: 2.2 ) in SD examinations $(p<0.001)$. Results of dose simulation with $z$-axis reduction to the range of the clavicula displayed a significantly lower radiation exposure with a median simulated ED of $0.1 \mathrm{mSv}$ (IQR: 0.0 ) in the LD group vs. $0.9 \mathrm{mSv}$ (IQR: 0.6) in the SD group $(p<0.001)$ (Table 2$)$.

\section{Image quality assessment}

Median image quality in SD imaging was 4 (IQR: 0). The image quality in LD imaging was rated significantly lower, with a median of 3 (IQR: 1$)$ by both readers $(p<0.001$ for both readers). There was almost perfect agreement between both readers concerning image quality assessment, with a value of 0.83 ( $p<0.001$; Table 3$)$. There was no significant difference regarding the presence of artifacts $(p=0.517)$. Both readers found one motion artifact in the same LD examination while no artifact was found in SD imaging. Figures 2, 3, 4, and 5 display an example for LD and SD imaging.

\section{Ossification stages and confidence levels of the whole study group}

There was a significant age difference between the LD group with $13.7 \pm 4.7$ years vs. $24.5 \pm 3.4$ years in the SD group $(p<0.001)$. The most often ossification stage in LD examinations was stage 1 in both readers (95 vs. 96 cases) while the SD group exhibited most often stage 4 in both readers (28 vs. 32 cases). There was no significant difference between both readers regarding the ossification stages $(p=0.405)$. Spearman's correlation between both radiologists was 0.985 $(p<0.001)$. Overall, there was almost perfect agreement between both readers with a kappa of $0.934(p<0.001)$. The analysis of the LD group only resulted in a Spearman's correlation of 0.975 and in an inter-reader agreement of 0.950 (both $p<0.001$ ). In SD imaging, Spearman's correlation was 0.913
Table 3 Correlation and interreader variability analysis

\begin{tabular}{|c|c|c|c|c|}
\hline & \multicolumn{2}{|c|}{ Spearman's correlation } & \multicolumn{2}{|c|}{ Inter-reader variability } \\
\hline & Correlation & $p$ value & Kappa & $p$ value \\
\hline Image quality $(n=207)$ & 0.913 & $<0.001$ & 0.831 & $<0.001$ \\
\hline Low-dose $(n=146)$ & 0.809 & $<0.001$ & 0.728 & $<0.001$ \\
\hline Standard-dose $(n=61)$ & 1 & & 1 & \\
\hline Ossification stages $(n=207)$ & 0.985 & $<0.001$ & 0.934 & $<0.001$ \\
\hline Low-dose $(n=146)$ & 0.975 & $<0.001$ & 0.950 & $<0.001$ \\
\hline Standard-dose $(n=61)$ & 0.913 & $<0.001$ & 0.855 & $<0.001$ \\
\hline Subset $2 \mathrm{a}-3 \mathrm{c}(n=65)$ & 0.986 & $<0.001$ & 0.925 & $<0.001$ \\
\hline Low-dose $(n=45)$ & 0.970 & $<0.001$ & 0.914 & $<0.001$ \\
\hline Standard-dose $(n=20)$ & 0.986 & $<0.001$ & 0.938 & $<0.001$ \\
\hline
\end{tabular}




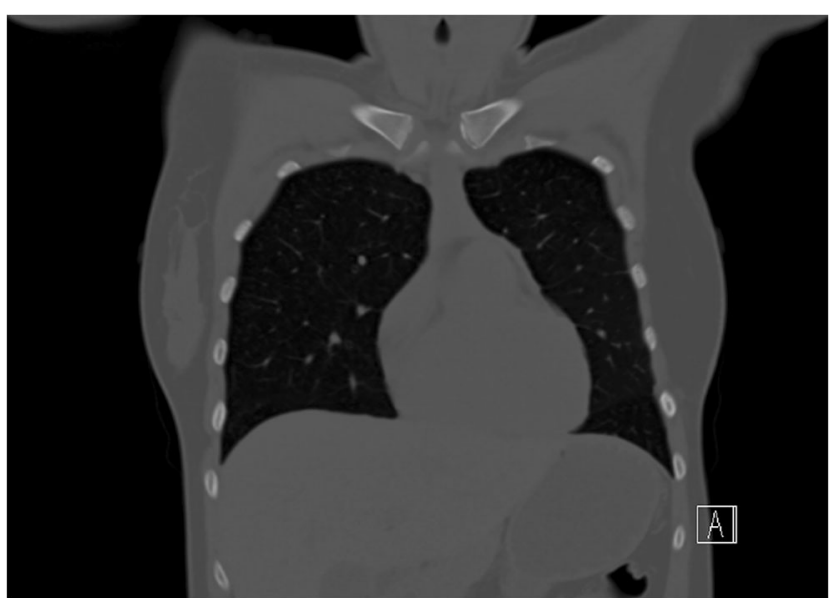

Fig. 2 Coronal view of a chest $\mathrm{CT}$ using a standard-dose imaging proto$\mathrm{col}(120 \mathrm{kV} /$ reference $100 \mathrm{mAs}$; effective dose $6.1 \mathrm{mSv})$. The ossification stage was classified as $2 \mathrm{c}$

and the inter-reader agreement analysis showed a kappa of 0.855 (both $p<0.001$; Table 3).

Median confidence levels of both readers were significantly higher in LD imaging with 4 (IQR: 0 ) vs.4 (IQR: 1 ) in SD imaging ( $p=0.035$ for reader $1, p=0.021$ for reader 2 ; Table 4).

\section{Ossification stages and confidence levels of the subset $2 a-3 c$}

A further analysis of a subset of 65 examinations with ossification stages $2 \mathrm{a}-3 \mathrm{c}$ was performed as these stages are the most difficult ones to differentiate. The mean patient age in this subset was $17.7 \pm 2.1$ years in LD imaging ( 45 cases) and $21.4 \pm 3.0$ years in SD imaging (20 cases). There was no significant difference between both radiologists regarding the ossification stages $2 \mathrm{a}-3 \mathrm{c}(p=0.317)$. Spearman's correlation of the ossification stages between both readers was 0.986 $(p<0.001)$. Although a slight decrease in the inter-reader agreement could be observed, it was still almost perfect with 0.925 ( $p<0.001$; Table 3). Median confidence level of reader 1 was 4 (IQR: 1) in LD imaging and 3.5 (IQR: 1) in SD imaging $(p=0.186)$. Median confidence level of reader 2 was 3 (IQR: 1) in LD imaging and 3 (IQR: 2) in SD imaging $(p=0.074$; Table 4$)$.

\section{Intra-reader variability assessment of the ossification stages}

A further subset of 32 pairs of examinations (25 pairs of LD imaging) which were performed within 100 days were used for intra-reader variability assessment of the ossification stages. Intra-reader agreement for both readers was 0.765 $(p<0.001)$.

\section{Discussion}

This study could show that LD CT imaging of the clavicula for forensic age determination in living subjects is feasible without loss of readers' confidence. Although the image quality was rated worse in the LD group, this did not affect the confidence levels of the readers regarding age estimation.

This is to our knowledge the first study investigating the feasibility of LD scans of the clavicula for forensic age estimation. As forensic imaging is carried out for medicolegal issues only, utmost efforts for radiation exposure reduction are necessary. It was shown previously that medical imaging using Xrays can cause DNA damage [17]. This is especially relevant as children and adolescents are more sensitive to ionizing radiation due to biological factors [18]. Additionally, adolescents exposed to radiation are more likely to develop cancer than adults due to their higher life expectancy and higher rates of cell division [19-21]. Large epidemiological studies have shown previously that patients who were exposed to CT examinations during their childhood exhibit higher cancer rates [22-24]. However, it is difficult to further specify the exact attributable risk of X-ray examinations due to the complexity of populationbased studies and the inherently associated confounders $[22$, $25,26]$.

These above mentioned issues strengthen the importance of radiation reduction. In our study, two different LD imaging

Fig. 3 Magnification of the medial clavicular joint of Fig. 2 (standard-dose)

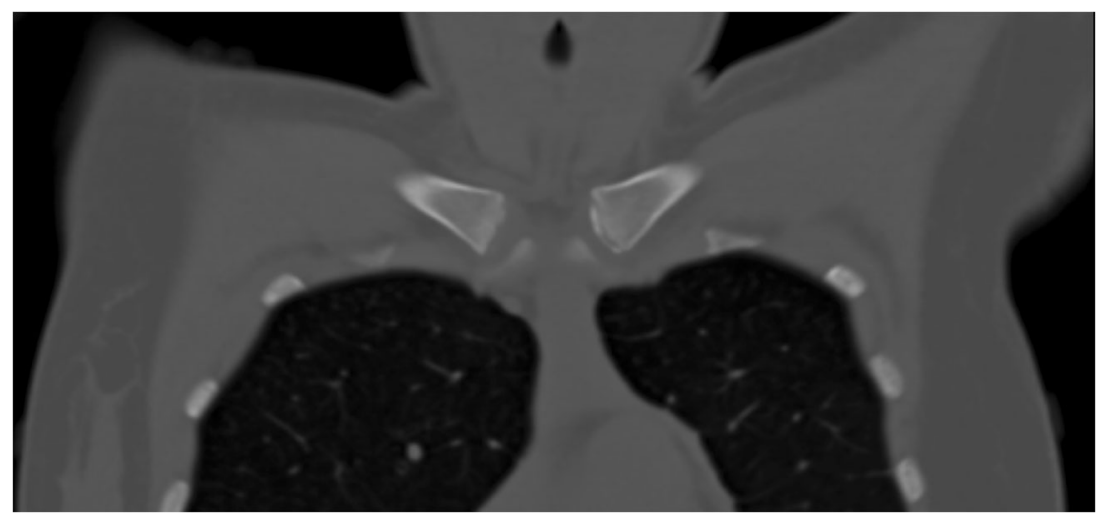




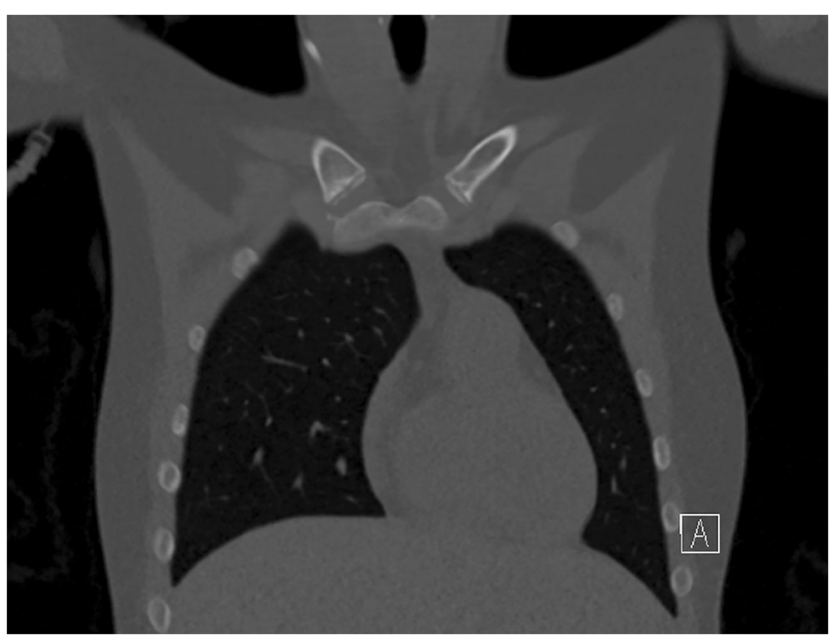

Fig. 4 Coronal view of a chest CT using a low-dose imaging protocol (Sn100/reference $96 \mathrm{mAs}$; effective dose: $0.4 \mathrm{mSv}$ ). The ossification stage was classified as $2 \mathrm{c}$

approaches were followed which resulted in a median radiation exposure in LD imaging of the clavicula of $0.1 \mathrm{mSv}$. This corresponds to an equivalent of approximately 2 weeks of natural background radiation [18]. Therefore, the slightly higher radiation dose compared with conventional radiography can be considered tolerable due to the higher robustness and reliability of CT data $[13,14]$. Although it was previously shown that also MRI of the medial clavicular epiphysis can be used for age estimation, the exact role and reliability of MRI remain still unclear in this area [27].

The high inter-reader agreement between both readers with different levels of experience in our study indicates that forensic age determination via CT of the clavicula seems to be a manageable challenge. Therefore, the application of LD CT imaging of the clavicula should not be reserved for highly specialized centers, only. Similar to the analysis of the skeletal bones of the left hand using the common radiographic atlas of skeletal development by Greulich and Pyle, bone age classification via CT should become a standard procedure [1].
Table 4 Confidence analysis depending on the imaging protocol

\begin{tabular}{llll}
\hline & Low-dose imaging & Standard-dose imaging & $p$ value \\
\hline \multicolumn{2}{l}{ Confidence level; median (IQR) } \\
Reader 1 & $4(0)$ & $4(1)$ & 0.035 \\
Reader 2 & $4(0)$ & $4(1)$ & 0.021 \\
Subset 2a-3c analysis; median (IQR) & \\
Reader 1 & $4(1)$ & $3.5(1)$ & 0.186 \\
Reader 2 & $3(1)$ & $3(2)$ & 0.074 \\
\hline
\end{tabular}

$I Q R$ interquartile range

It is striking that the analysis of the whole study group revealed higher confidence levels for the LD group compared with SD imaging. This is probably due to the high number of patients with the easily identifiably ossification stage 1 in the LD group. Therefore, a further subset analysis of the stages $2 \mathrm{a}-3 \mathrm{c}$ was performed which showed no significant difference of confidence between LD and SD imaging. This is especially important as the stages $2 \mathrm{a}-3 \mathrm{c}$ are the most difficult to differentiate. Additionally, the exact classification of adolescents within this range of age plays a vital role for further implications resulting from the categorization as juvenile or adult delinquent.

\section{Limitations}

Our study was a monocentric, retrospective study using mostly modern dual-source generation scanners equipped with all technical benefits such as tin filtration and iterative reconstruction algorithms. A further limitation that merits consideration is the different group size of patients in LD and SD imaging. Additionally, the patients in the SD group were slightly older, as LD imaging protocols were preferably applied in very young patients. Most patients suffered from a malignant primary disease with a possible effect on the bone age. However,
Fig. 5 Magnification of the medial clavicular joint of Fig. 4 (low-dose)

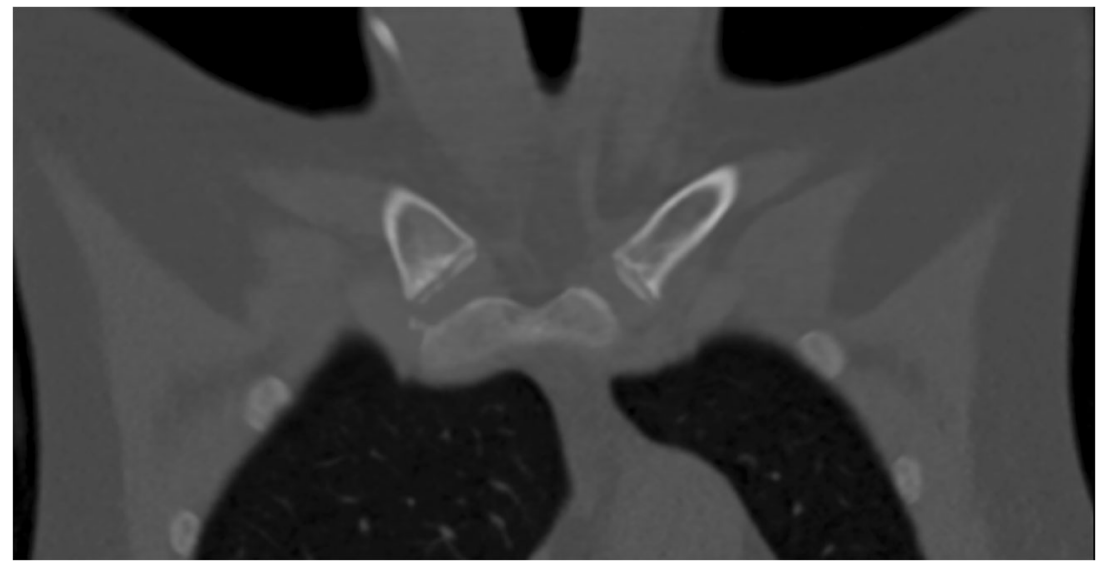


the aim of this study was not to compare bone age with chronological age but to analyze the feasibility and impact of a LD imaging protocol. Additionally, CT imaging is no primary diagnostic tool in this young patient cohort. Consequently, almost no CT studies were available from patients without severe primary disease, though further prospective studies are necessary to confirm these initial results on a broader spectrum of patients and different scanner architectures.

\section{Conclusions}

The results of this study indicate that LD CT imaging of the medial clavicular joint for age estimation is possible without loss of readers' confidence. By restricting the scanning area to the clavicula only, the radiation exposure can be reduced to an equivalent of approximately 2 weeks of natural background radiation.

Funding information Open Access funding enabled and organized by Projekt DEAL.

\section{Compliance with ethical standards}

Guarantor The scientific guarantor of this publication is Dr. Ilias Tsiflikas.

Conflict of interest The authors of this manuscript declare no relationships with any companies, whose products or services may be related to the subject matter of the article.

Statistics and biometry No complex statistical methods were necessary for this paper.

Informed consent Only if the study is on human subjects, written informed consent was waived by the Institutional Review Board.

Ethical approval Institutional Review Board approval was obtained.

\author{
Methodology \\ - retrospective \\ - diagnostic or prognostic study \\ - performed at one institution
}

Open Access This article is licensed under a Creative Commons Attribution 4.0 International License, which permits use, sharing, adaptation, distribution and reproduction in any medium or format, as long as you give appropriate credit to the original author(s) and the source, provide a link to the Creative Commons licence, and indicate if changes were made. The images or other third party material in this article are included in the article's Creative Commons licence, unless indicated otherwise in a credit line to the material. If material is not included in the article's Creative Commons licence and your intended use is not permitted by statutory regulation or exceeds the permitted use, you will need to obtain permission directly from the copyright holder. To view a copy of this licence, visit http://creativecommons.org/licenses/by/4.0/.

\section{References}

1. Greulich WW, Pyle SI By WilliamWalter Greulich and S. Idell Pyle. (1950) XIII + 190 pages, illustrated. \$10.00. Stanford University Press, Stanford, California. 108:335-336

2. Tise M, Mazzarini L, Fabrizzi G, Ferrante L, Giorgetti R, Tagliabracci A (2011) Applicability of Greulich and Pyle method for age assessment in forensic practice on an Italian sample. Int J Legal Med 125:411-416

3. Schmeling A, Grundmann C, Fuhrmann A et al (2008) Criteria for age estimation in living individuals. Int J Legal Med 122:457-460

4. Choi JA, Kim YC, Min SJ, Khil EK (2018) A simple method for bone age assessment: the capitohamate planimetry. Eur Radiol 28: 2299-2307

5. De Tobel J, Hillewig E, de Haas MB et al (2019) Forensic age estimation based on T1 SE and VIBE wrist MRI: do a one-fits-all staging technique and age estimation model apply? Eur Radiol 29: 2924-2935

6. Li Y, Huang Z, Dong X et al (2019) Forensic age estimation for pelvic X-ray images using deep learning. Eur Radiol 29:2322-2329

7. Vieth V, Schulz R, Heindel W et al (2018) Forensic age assessment by 3.0T MRI of the knee: proposal of a new MRI classification of ossification stages. Eur Radiol 28:3255-3262

8. Kreitner KF, Schweden F, Schild HH, Riepert T, Nafe B (1997) Computerized tomography of the epiphyseal union of the medial clavicle: an auxiliary method of age determination during adolescence and the 3d decade of life? Rofo 166:481-486

9. Kreitner KF, Schweden FJ, Riepert T, Nafe B, Thelen M (1998) Bone age determination based on the study of the medial extremity of the clavicle. Eur Radiol 8:1116-1122

10. Kellinghaus M, Schulz R, Vieth V, Schmidt S, Schmeling A (2010) Forensic age estimation in living subjects based on the ossification status of the medial clavicular epiphysis as revealed by thin-slice multidetector computed tomography. Int J Legal Med 124:149-154

11. Schmeling A, Schulz R, Reisinger W, Muhler M, Wernecke KD, Geserick G (2004) Studies on the time frame for ossification of the medial clavicular epiphyseal cartilage in conventional radiography. Int J Legal Med 118:5-8

12. Schulz R, Muhler M, Reisinger W, Schmidt S, Schmeling A (2008) Radiographic staging of ossification of the medial clavicular epiphysis. Int J Legal Med 122:55-58

13. Wittschieber D, Ottow C, Vieth V et al (2015) Projection radiography of the clavicle: still recommendable for forensic age diagnostics in living individuals? Int J Legal Med 129:187-193

14. Wittschieber D, Ottow C, Schulz R et al (2016) Forensic age diagnostics using projection radiography of the clavicle: a prospective multi-center validation study. Int J Legal Med 130:213-219

15. Kellinghaus M, Schulz R, Vieth V, Schmidt S, Pfeiffer H, Schmeling A (2010) Enhanced possibilities to make statements on the ossification status of the medial clavicular epiphysis using an amplified staging scheme in evaluating thin-slice CT scans. Int J Legal Med 124:321-325

16. Muhler M, Schulz R, Schmidt S, Schmeling A, Reisinger W (2006) The influence of slice thickness on assessment of clavicle ossification in forensic age diagnostics. Int J Legal Med 120:15-17

17. Nguyen PK, Lee WH, Li YF et al (2015) Assessment of the radiation effects of cardiac CT angiography using protein and genetic biomarkers. JACC Cardiovasc Imaging 8:873-884

18. United Nations Scientific Committee on the Effects of Atomic Radiation (2008) Sources and effects of ionizing radiation. https:// www.unscear.org/docs/reports/2008/11-80076_Report_2008 Annex_D.pdf

19. Brenner DJ (2002) Estimating cancer risks from pediatric CT: going from the qualitative to the quantitative. Pediatr Radiol 32:228 221 discussion 242-224 
20. Hall EJ (2002) Lessons we have learned from our children: cancer risks from diagnostic radiology. Pediatr Radiol 32:700-706

21. Brenner D, Elliston C, Hall E, Berdon W (2001) Estimated risks of radiation-induced fatal cancer from pediatric CT. AJR Am J Roentgenol 176:289-296

22. Rigsby CK, McKenney SE, Hill KD et al (2018) Radiation dose management for pediatric cardiac computed tomography: a report from the Image Gently 'Have-A-Heart' campaign. Pediatr Radiol 48:5-20

23. Pearce MS, Salotti JA, Little MP et al (2012) Radiation exposure from CT scans in childhood and subsequent risk of leukaemia and brain tumours: a retrospective cohort study. Lancet 380:499-505

24. Mathews JD, Forsythe AV, Brady Z et al (2013) Cancer risk in 680, 000 people exposed to computed tomography scans in childhood or adolescence: data linkage study of 11 million Australians. BMJ 346:f2360
25. Hauptmann M, Meulepas JM (2012) CT scans in childhood and risk of leukaemia and brain tumours. Lancet 380:1736 author reply 1736-1737

26. Zopf DA, Green GE (2012) CT scans in childhood and risk of leukaemia and brain tumours. Lancet 380:1735-1736 author reply 1736-1737

27. Hillewig E, De Tobel J, Cuche O, Vandemaele P, Piette M, Verstraete K (2011) Magnetic resonance imaging of the medial extremity of the clavicle in forensic bone age determination: a new four-minute approach. Eur Radiol 21:757-767

Publisher's note Springer Nature remains neutral with regard to jurisdictional claims in published maps and institutional affiliations. 